accept the heavy extra work and surrender of their freedom involved; and the influencing of public opinion against the scheme by those who did not wish to co-operate. Moreover, those who believed the scheme was workable did not express themselves strongly enough, and no central authority was appointed in reception areas to deal with the problems which arose, to give a lead to parents and hosts who were uncertain in their attitude and to undertake active propaganda to. influence public opinion in favour of the scheme. Such a central authority in each district ready to offer advice on all difficult problems, particularly psychological ones of behaviour, could undoubtedly have smoothed out many of the difficulties and led to the children remaining in the safe areas. So far as there have been breakdowns in the scheme, the administrative machinery would appear to be at fault rather than any fundamental weakness in the ties which bind our community together, or absence of public spirit on the part of hosts or parents of evacuated children. If the authorities concerned had used the services of tutors qualified in medical psychology or carried out a short research before the scheme was drawn up, many mistakes would have been avoided.

The report also stresses the possibility of basing action on precise knowledge with the development of social psychology. It directs attention to the need for extended research on enuresis and its treatment, the widespread existence of which was indicated in the inquiry. The scheme also demonstrated that the general standards of cleanliness and hygiene leave much to be desired, and the appreciation of the value of education is much lower in a certain group of the population than might have been supposed. Much could be done to correct this attitude by strengthening the school medical service, and the use of new means of education and propaganda.

In regard to the apportionment of responsibility for individual failures, the inquiry showed that 60 per cent of the parents were to blame for taking their children home without justifiable cause, 30 per cent of the hosts sent the children away because they did not wish to co-operate and only 10 per cent of the children returned because they were ill-behaved or fretting.

\title{
LIMITATION OF TRANSFORMER NOISE
}

\begin{abstract}
$\mathrm{T}$ RANSFORMERS are particularly suited for outside use in outside distribution of electricity, owing to the facility with which they can be installed and the saving effected, but if they are a nuisance to dwellers in their neighbourhood, something has to be done immediately to suppress their noise. Noise prevention is a matter for co-operative investigation, and this was the reason why the Electrical Research Association (E.R.A.) formed a committee in 1934, composed of gas, electric and water engineers, under the chairmanship of Prof. B. O. Kapp, to consider the best means of eliminating this trouble. Definite contributions have been made to our knowledge by this committee, and by papers and discussions on the subject contributed to the engineering societies. An interesting paper on the limitation of transformer noise was contributed to the Institution of Electrical Engineers on March 28, the authors being B. G. Churcher and A. J. King, of the MetropolitanVickers Electrical Co., Ltd.

Before considering means for reducing noise to allowable proportions, we should be able to determine its value both as regards quantity and quality. In the case of transformers, the latter is not so important as in the case of some kinds of machinery in which the sound varies in pitch. Nevertheless, the annoyance which can be caused by transformer hum is very real.
\end{abstract}

It is axiomatic that trouble should, if possible, be eliminated at its source. The authors and those who took part in the discussion on their paper showed there is very little to be gained by altering the design of transformers within permissible commercial limits. Dr. Swaffield has recently shown that the main cause of transformer noise is magnetostriction, that is, vibration due to change in length and in the area of the core that occur together, under the influence of the impressed magnetic field. The old idea that tightening core bolts and clamping could effect appreciable improvement has been shown to be wrong. Attempts to limit the effects of magneto-striction by reducing the density of the magnetic flux yield only very slight relief at a great cost.

The Electrical Review of April 5 gives a good illustration of the material values of the study that has been expended on this subject. When the $\mathbf{7 5 , 0 0 0} \mathrm{kva}$. transformers were installed at Fulham Power Station about three and a half years ago, all means were taken to avoid being a cause of offence. The transformers were run at a low flux density and the tanks rested on rubber pads. Each transformer was housed in a brick chamber without openings. This last precaution was probably much the most effective.

Many think that efforts to obtain a silent transformer will probably be fruitless. Attenuation of unavoidable sound offers the most promising field of attack. Hence the authors put forward, as a less costly alternative to bricking-in, where conditions would otherwise make that necessary, a scheme of barriers within a larger tank which would absorb both radial and longitudinal sound waves. Even apart from the dominant consideration of the background level of noise, almost every case has to be considered on its merits. Distance and structure of the surrounding houses are principal factors, but even here resonance sometimes gives rise to curious phenomena.

The first need appears to be to secure a standard attenuation acceptable in ordinary. conditions, and then to provide means to predict with certainty the additional attenuation required to meet the peculiarities of local circumstances. In addition, the establishment of noise-levels regarded as tolerable would be helpful, if only because (since psychology enters into the equation) objection to constituted standards would appear unreasonable. It may be fairly claimed that research on present lines augurs well for an economical solution of the noise problem, without departing from the well-established principles of transformer design. 